\title{
Crickets as bio-inspiration for MEMS-based flow-sensing.
}

\author{
Gijs Krijnen ${ }^{1, \mathrm{a}, *}$, Harmen Droogendijk ${ }^{1, \mathrm{~b}}$, Ahmad Dagamseh ${ }^{1, \mathrm{c}}$, \\ Ram Jaganatharaja ${ }^{1, \mathrm{~d}}$ and Jérôme Casas $^{2, \mathrm{e}}$ \\ ${ }^{1} \mathrm{MESA}^{+}$Research Institute, University of Twente, Enschede, The Netherlands \\ ${ }^{2}$ Institut de Recherche en Biologie de I'Insecte, Universitf́ de Tours, France \\ \{aijs.krijnen, bh.droogendijk\}@utwente.nl, cahmad.dagamseh@gmail.com, \\ dram.kottumakulal@asml.com, fjerome.casas@univ-tours.fr, *corresponding author
}

Keywords: Flow, sensor, hair, cricket, fish, viscous coupling, parametric effects

\begin{abstract}
MEMS offers exciting possibilities for the fabrication of bioinspired mechanosensors. Over the last years we have been working on cricket inspired hair-sensor arrays for spatio-temporal flowfield observations (i.e flow-camera) and source localization. Whereas making flow-sensors as energy efficient as cricket hair-sensors appears to be a real challenge we have managed to fabricate capacitively interrogated sensors with sub-millimeter per second flow sensing thresholds, to use them in lateral line experiments, address them individually while in arrays, track transient flows, and use nonlinear effects to achieve parametric filtering and amplification. In this research insect biologists and engineers have been working in close collaboration, generating a bidirectional flow of information and knowledge, beneficial to both. E.g. where the engineering has greatly benefitted from the insights derived from biology and biophysical models, the biologists have taken advantage of MEMS structures allowing for experiments that are hard to do on living material.
\end{abstract}

\section{Introduction}

The filliform hairs of many insects, spiders and other invertebrates are among the most delicate and sensitive flow sensing cells: they measure displacement of less than a hydrogen diameter (sensitivity ca. $10^{-10} \mathrm{~m}=1 \AA$ ) and react to flow speed down to $30 \mu \mathrm{m} / \mathrm{s}$. If one considers the energy needed to trigger a cell reaction, one finds that they react with a thousandths of the energy contained in a photon, so that they surpass photoreceptors in energy sensitivity. In fact, these mechanoreceptors work at the thermal noise level [1]. These hairs pick up air motion, implying that they are measuring both the direction and speed of air flow, in contrast to pressure receivers, i.e. ears. Since several decades the biomechanics of the filiform hairs have been studied with care by several groups worldwide, based on the analogy with a single degree of freedom inverted pendulum (see e.g. the review of hair biomechanics in [2]).

Among insects, mainly cockroaches and crickets have been studied, because their air flow sensitive hairs are put on two antenna-like appendages, the cerci (cercus in singular). Insect hairs usually have a high aspect ratio, with a length of a few hundreds of microns up to $2 \mathrm{~mm}$, and with a diameter of less than a dozen microns (Fig. 1). Their longitudinal shape is conical which has been found to have an influence on the drag forces. Hairs and sockets are ellipsoidal in cross-section, which leads to a preferential direction of movement. The base of the hairs is complex, and its mechanics poorly understood. In crickets, only a single sensory cell is below the hair shaft (Fig. 1).

Single hairs, or groups of hairs, are not placed at random on the body [3, 4]. For the same reasons that the exact shape of hairs as well as their relative position within a group both have been most likely molded by natural selection, the position of the hairs on the sensory organ must obey to strong selection. This aspect of mechanosensory research is however badly neglected. As for the positions along the cercus, the presence of a potential acoustic fovea (i.e. a location with particularly high acuity) at the base of the cercus has been hinted at, not only due to the highest hair density in this region, but also because it corresponds to the region with the largest flow velocities, the cercus being the largest there. Putting hairs radially around the cercus enables crickets also to pick up transversal flows, whose 


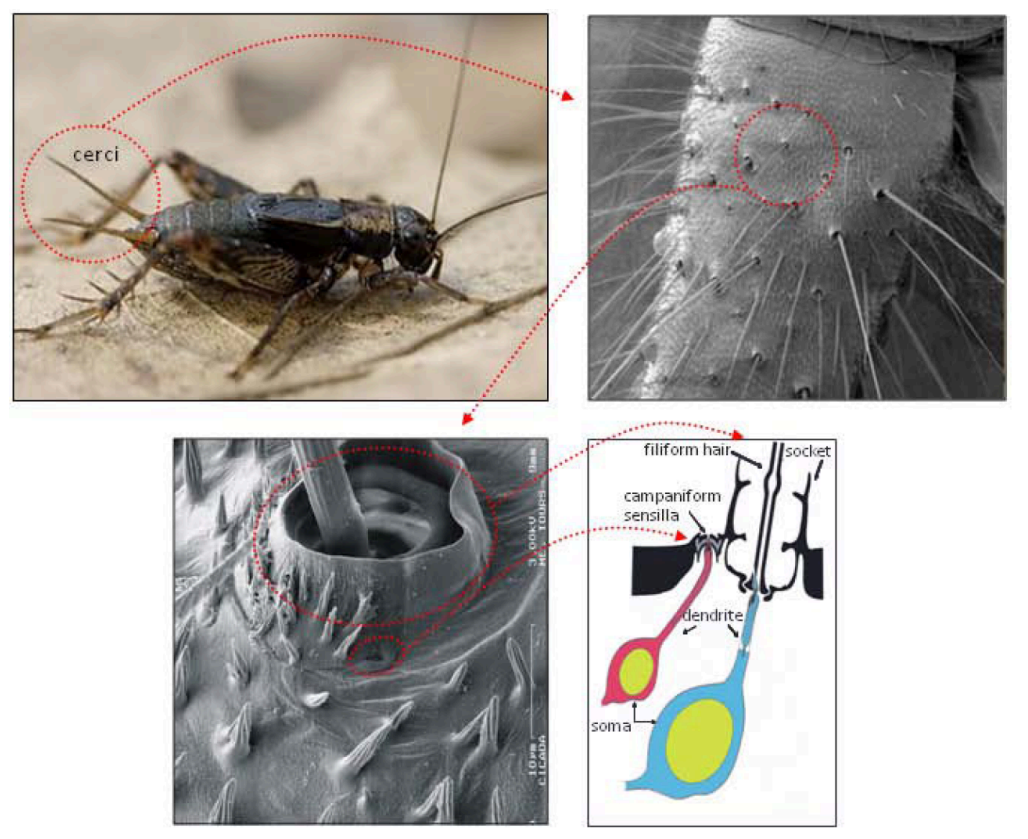

Fig. 1: Illustration of the hair-sensory system of crickets.

peak flow velocities are larger than in the situation where the hairs would be placed on a flat surface; in the latter case, hairs are submitted to longitudinal flow with lower peak velocities [5]. In summary, where you put your sensors relative to your body geometry matters a lot.

An action potential triggered by a moving hair ends up in the terminal abdominal ganglion (TAG), a local neuronal processing unit. Information from all the hairs, as well as from other sensors, converges there and is processed by interneurons. The compression of information at this stage is huge: the about 1500 afferent neurons of hairs are connected to only some 20 interneurons [6]. The fact that invertebrates possess few large, singly identifiable neurons enabling comprehensive mapping and repeated recordings of activities is a unique asset which explains the interest in such exotic systems. Information coming from the central brain as well as from the higher ganglia also descends into the TAG. Once processed, the combined information moves up quickly towards higher neuronal centres, in particular the ganglia in which the hindleg movements are being decided. This local feedback loop, with little input from the main brain, enables the animal to process vital information and act accordingly very quickly. As so often with invertebrates, what can be processed locally should be done so, a distributed processing scheme which explains why biomimetism has so much to gain from this group of animals.

The last level of integration is behaviour, and flow sensing is known to be of importance in predator and prey perception, sexual selection and most likely other context, such as noticing its own speed and movement. Predator avoidance is obviously a major selection force, where speed is of paramount importance. Jumping or running away is the behavior which is elicited using appropriate stimuli. The cricket possesses in the TAG an internal map of the direction of the stimuli from the outside world and the geometric computation of the direction of incoming flow by the cercus is one of the nicest case study of spatial representation [6]. Computing the speed of an approaching predator is also carried out by the TAG, and has been only recently established using appropriate stimuli [41]. Where to jump is a different question, in which directing stimuli and other conditions intervene.

Natural selection acts along the full chain of information transfer, from acquisition and processing, up to actuation. This is important to restate in a biomimetic context, as the extreme sensitivity on the biomechanical side of the hair shaft, which has been almost the exclusive focus of the engineer attention, could be otherwise lost into an inefficient sequence of information transfer. As of today, we have however very little information about the constraints acting on the different parts of the chain, and hence no idea about their optimisation levels. 


\section{Hair-sensors}

Flow as information source. Whereas for humans (mammals) flow may not seem a very information rich modality for probing the environment, nature seems to have numerous examples of species that exactly do this. To put this in perspective it may be helpful to look at the fields produced by a harmonically moving sphere (dipole), which, with some simplifications, may resemble more natural sources such as wing-beats of flying insects or tail movements of fish. Obviously the fields produced by a dipole entail both pressure and flow fields. Depending on the medium, e.g. water or air, in which the dipole resides, and more specifically on the mediums compressibility, it may seem that pressure and flow could play comparably important roles, albeit that flow-fields inherently carry directional information (being vector-fields) whereas pressure is a scalar field only. How much this observation may be true at relatively long distances (for compressible media one could think of at least a few wavelengths away from the source) where the ratio of flow- and pressure fields becomes constant, near to the source flow-fields are comparatively "more present". This is shown in Fig. 2 left, where on-axis pressure and velocity, normalized to their respective values at $k \cdot r=1$ (with $k$ the wavenumber and $r$ the distance), are plotted as a function of normalized distance. Both the situations of compressible (green, blue) and incompressible (red) media, as calculated from the expressions in [7], are shown. Clearly, for $k \cdot r \leq 1$, i.e. distances smaller than the wavelength, there are only minor differences between compressible and incompressible theory, a fact that can be readily exploited when modeling relative complex aerodynamic predator-prey interactions [8].
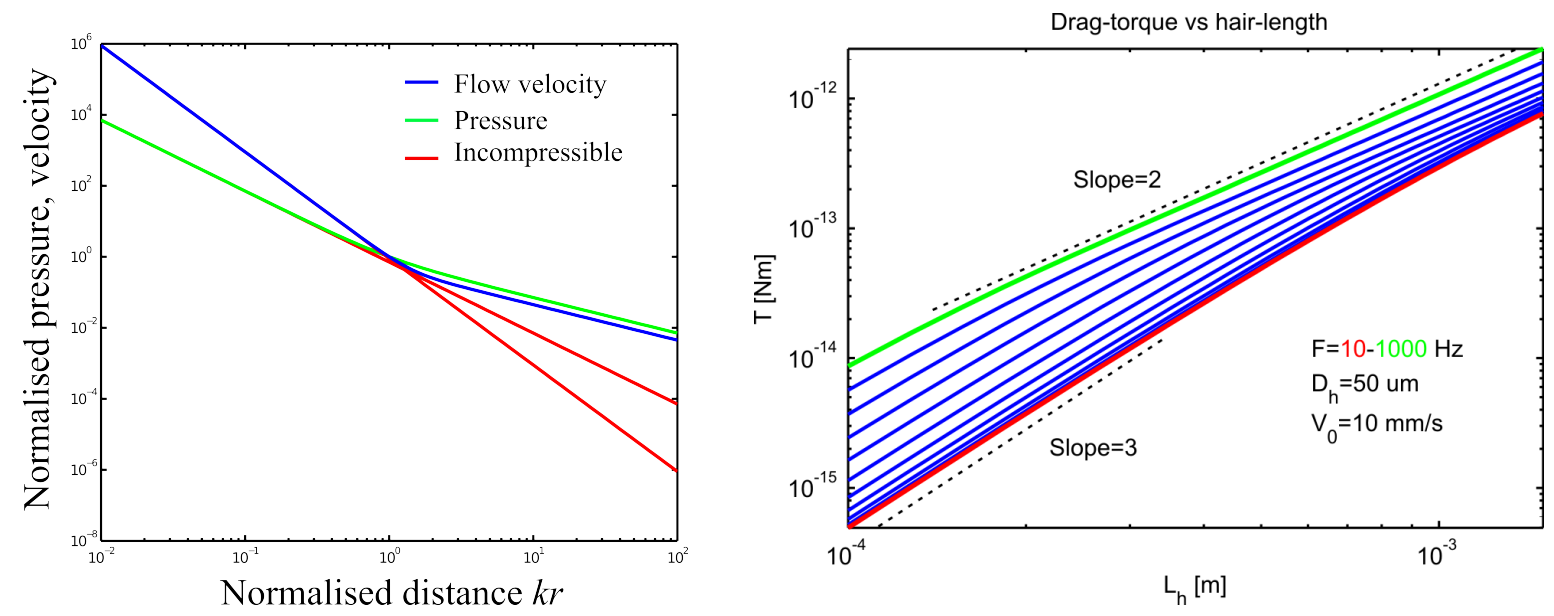

Fig. 2: Left: theoretical dipole-field as function of normalised distance. Right: drag-torque versus hair-length for harmonic air-flow of various frequencies as predicted by Stokes' equations.

The ratio between pressure and flow velocity (which would be the acoustic impedance for compressible media) equals $j \rho \omega r / 2$ ( $\rho$ being the density, $\omega$ the angular frequency) for $k \cdot r \ll 1$ indicating that pressure becomes comparatively small for shorter distances and lower frequencies. As an example for an interaction of a flying wasp with a wing beat of $\approx 150 \mathrm{~Hz}$ with, say, a caterpillar [9] the condition $k \cdot r=1$ corresponds with $r \approx 0.34 \mathrm{~m}$ a relative large distance as measured in body lengths of the animals.

Hair-sensor physics. There have been a few seminal papers (e.g. [10] and [30]) describing the interaction of harmonic flows with hairs. In general these flows have been assumed to be small which, in combination with the small hair diameters, yields rather small Reynolds $(R)$ numbers. Moreover, the frequencies are limited to a few $100 \mathrm{~Hz}$ causing the Strouhal number $\left(S_{t}=\omega d / 2 V_{r}\right)$ to be small as well. For a hair-diameter of $25-50 \mu \mathrm{m}$, an air-oscillation frequency of $250 \mathrm{~Hz}$ and a flow-velocity amplitude of $10 \mathrm{~mm} / \mathrm{s}, R$ varies between $0.008-0.016$ and $S_{t}$ between $1.96-3.92$ (for the flow around the hairs). The rather small Reynolds numbers and the large hair-length to hair-diameter ra- 
tio allow using the Stokes expressions for the drag-torque exerted by the air-flow on the hairs [12]. Further our artificial hair-sensors are mounted on flat substrates allowing the use of the Stokes expressions for harmonic flows along the hairs [10]. These expressions predict a viscous flow over an infinite substrate to be harmonic in time with zero flow-velocity and 45 degrees phase advance at the substrate interface and a boundary layer thickness $\left(\delta_{b}\right)$ proportional to the inverse of $\beta=\sqrt{\omega / 2 \nu}$ where $\nu$ is the kinematic viscosity $\left(1.79 \cdot 10^{-5} \mathrm{~m}^{2} / \mathrm{s}\right.$ for air at room-temperature) and $\omega$ is the radial frequency. As an example, at $100 \mathrm{~Hz}$ the boundary layer is roughly $0.5 \mathrm{~mm}$ thick. We have shown previously that the Stokes expressions can be usefully employed for our artificial hair-sensors [13]. Mechanically, the hair-sensor can be understood as a so-called inverted pendulum; a second-order rotational-mechanical system with moment of inertia $I$ due to the hair, a rotational stiffness $S$ and a rotational damping $R$. De air-flow generates a torque on the hair-shaft, primarily by viscous drag since at the velocities and geometries normally encountered pressure drag is small. Note that under most conditions artificial hairs can be assumed infinitely stiff and that the rotation angles are rather small (of the order of $1-10 \mathrm{mrad}$ amplitude per $\mathrm{m} / \mathrm{s}$ flow-velocity amplitude).
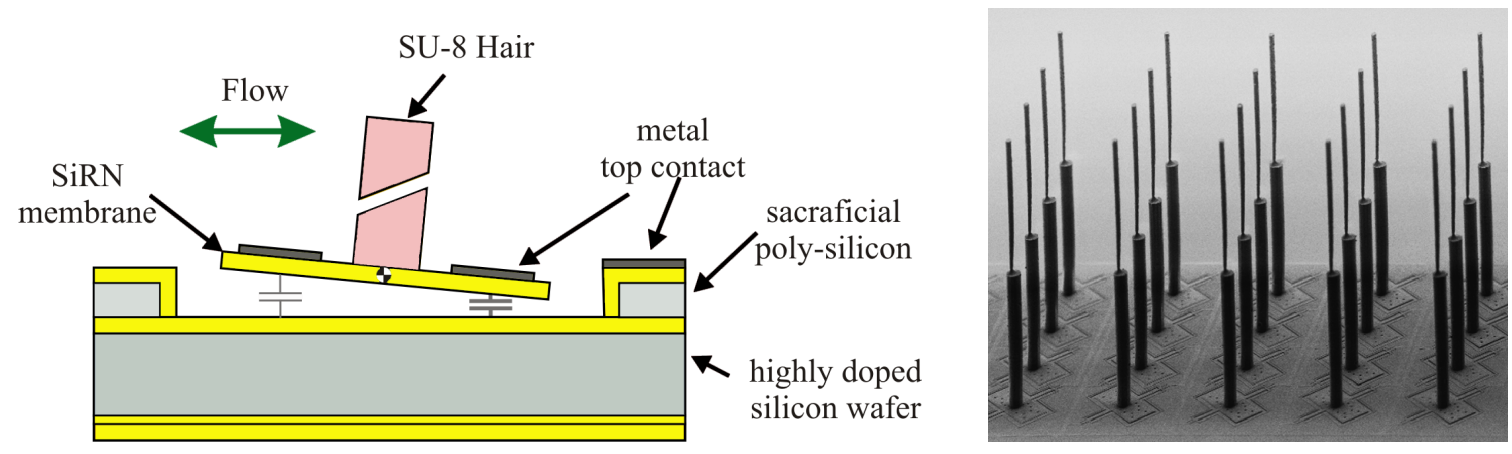

Fig. 3: Left: Schematic of the artificial hair-sensors using differential capacitive read-out. Right: Scanning Electron Micrograph (SEM) of a realized hair-sensor array.

Hair-sensor design. From a biological standpoint one may want to understand the entanglement of the geometric and physical parameters of the hair-sensor system as they are. However, from an engineering viewpoint things look slightly different since a) not all detail and interplay of all the involved parameters of the insects hair-sensor system are well-known (i.e. plain mimicking of the cricket cercal system is no option) and b) MEMS fabrication technology offers a latitude of size possibilities and material choices that only partly overlaps the natural system. Therefore the value of various design parameters need to be determined from other analyses.

A. Hair length and boundary layer. The length of the hairs $(L)$ plays a dominant role in the overall performance of the hair-sensors. Obviously, when exposed to a uniform flow the total drag torque on cylindrical hairs would increase proportional with the hair-length squared. However, due to the frequency depending boundary layer the drag-torque first increases with the third power of hair-length up to about $\delta_{b}$ and then follows a quadratic dependence (see Fig. 2, right). But at the same time the hair inertial moment $(I)$ is of order $O\left(L^{3}\right)$.

B. Hair diameter and viscous drag. When increasing the diameter of the hairs $(d)$ the resulting dragforce will increase as well. Using numerical evaluation of the Stokes expressions for drag-force shows that the dependence on diameter is weak in the range of interesting hair-diameters, of the order of $O\left(d^{1 / 3}\right)$. At the same time the hair moment of inertia is of order $O\left(d^{2}\right)$, negatively affecting the resonance frequency (i.e. bandwidth). Therefore it is beneficial to have thin hairs. Technologically it turns out to be rather difficult to make hairs with aspect ratios of more than about 10-20. We have tackled this problem by segmenting our artificial hairs with a lower part diameter of $50 \mu \mathrm{m}$ and a top part 
diameter of $25 \mu \mathrm{m}$, reducing the hair inertial moment by about $65 \%$ [14].

C. Torsional stiffness. Obviously, when looking for the largest rotation angle for any given drag-torque one may want to choose the lowest possible torsional stiffness $(S)$. But for given hair inertial moment a reduction of $S$ also leads to a reduction of the resonance frequency which is given by $\omega_{0}=\sqrt{S / I}$.

D. Damping. Damping of the hair-sensors comes in multiple forms. For the crickets the hair-sockets provide some torsional damping $(R)$ by visco-elastic material properties (see [15] for such material properties in spider hair mechano sensors) whereas for the artificial hair-sensors torsional damping is caused by both material as well as squeeze film damping due to the small gap between the siliconnitride plates and the substrate. On top of these damping contributions the hairs themselves incur damping by viscous forces when the hairs move relatively to the surrounding air. In the case of crickets the total damping seems to be appropriately controlled [45] by the organism yielding hairs that are approximately critically damped. It is hypothesized that mechanical impedance matching helps the sensors to obtain maximum energy from the surroundings [30]. On the other hand, on critically damping a second order system one also maximizes its agility to respond to (transient) flows [16]. Nevertheless, the evolutionary pressures driving the appropriate damping for cricket hair-sensors have not yet been identified. In the artificial hair-sensors, except for adding specific holes to the membranes to tailor the squeeze film damping, not much can be done to optimize the damping without far-reaching consequences for the fabrication technology.

E. Torsional spring material. The mechanical sensitivity of our hair-sensors is currently about 2 orders of magnitude less than those of crickets, primarily due to a much larger rotational stiffness: $1.5 \cdot 10^{-11} \mathrm{Nm} / \mathrm{rad}$ for crickets versus $4.85 \cdot 10^{-9} \mathrm{Nm} / \mathrm{rad}$ for our sensors. But reducing the torsional stiffness comes with two difficulties. In order to conserve bandwidth the moment of inertia of the hairs needs to be further reduced. The second complication is that the suspension beams provide torsional- $(S)$ as well as vertical stiffness $(K)$. Both decrease with increasing length $l$ but $S$ decreases with $O\left(l^{-1}\right)$ whereas $K$ decreases with $O\left(l^{-3}\right)$. The result is that a large rotational compliance combined with a large vertical stiffness can only be obtained using compliant materials, i.e. with low Youngs modulus and appropriate beam-cross-sections. A nice reference to nature despite the fact that our torsional suspension and the cricket hair-sockets have little in common.

F. Figure of Merit. Optimization of our hair-sensors has been driven by a Figure of Merit (FoM), basically being the product of mechanical responsivity and bandwidth [17]: $F o M=\sqrt{L / \rho S d^{4 / 3}}$. This has emphasized what could be learned directly from observation of cricket hair-sensors, i.e. that hairs should be long and thin, and mounted on very compliant suspensions. However, with respect to damping the optimum damping factors still need to be identified. Compared to crickets, for a $1 \mathrm{~mm}$ long hair the FoM of our hair-sensors is about a factor of 70 smaller due to the larger rotational stiffness and thicker hairs.

G. Capacitive read-out. The angular rotations induced by harmonic flows normally encountered are rather small: on the order of $1 \mathrm{mrad} /(\mathrm{mm} / \mathrm{s})$. Therefore the capacitive read-out needs to be judiciously implemented. Our hair-sensors are based on a differential read-out, using a $1 \mathrm{MHz}$ interrogation signal, a charge amplifier and a multiplier to retrieve the base-band information. Since parasitics due to bond-pads and wires are relatively large the fractional capacitance changes, which ultimately determine the sensitivity of the sensor, need to be optimized. Because the sensor's membrane area close to the rotational axis does not generate much capacitance change the membrane should primarily be long. Also, the smaller the effective gap between the capacitor electrodes, the larger the effect. Eventually the fractional capacitance change is given by $\partial C / \partial \alpha \cdot 1 / C=l / d$, giving clear directions for optimization. Early generations of our sensors were affected with stress-induced upward curvature 

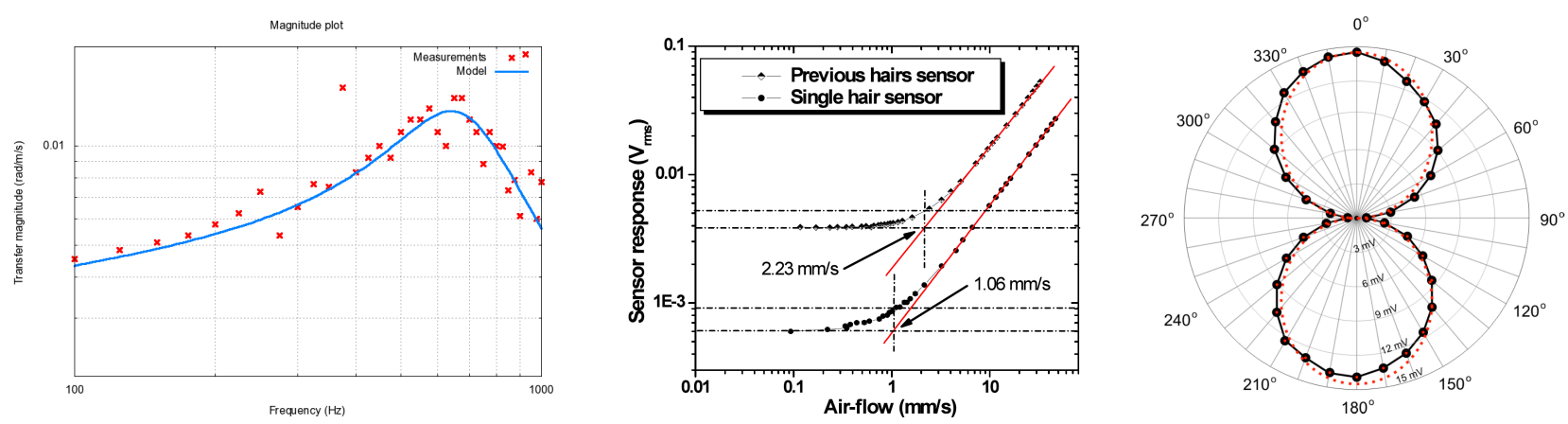

Fig. 4: Frequency response of a MEMS-based hair-sensor (left), its single-hair threshold at $250 \mathrm{~Hz}$ (middle, lower curve) and directivity (right). (Unpublished data and [18]).

of the membranes, negatively influencing the capacitive sensitivity. In later generations aluminum is used as electrode material since it has a high electrical conductivity (and therefore the layer can be thin), has a low Youngs modulus (thus will cause relative little bending) and can be deposited at low temperatures (reducing residual thermal stress).

The latest generation of our artificial hair-sensors is based on silicon-on-insulator (SOI) technology (see Fig. 9 left) which helps to reduce parasitic capacitances. The performance of this type of sensors is shown in Fig. 4 where results are displayed for a single hair-sensor. The threshold flow-amplitude value is at about $1.00-1.25 \mathrm{~mm} / \mathrm{s}$ for frequencies between $100-400 \mathrm{~Hz}$, which is currently determined by electronics noise (thermal-mechanical noise is predicted to be more than two order of magnitude smaller). There is also a clear directivity pattern, closely matching a theoretically ideal figure of 8 [18].

Optimisation of hair geometry Artificial hairs on top of the sensor membranes are the mechanical interfaces that cause the flow information to result in membrane rotations inducing capacitance changes which are eventually transformed into equivalent electrical signals.

A. Importance of hair shape. For the effective operation of our artificial hair sensors, the shape of the SU-8 hair plays a central role. The hair geometry serves two basic purposes: (i) it determines the amount of flow-induced drag-torque acting upon the hair and (ii) it contributes to the mass moment of inertia, which determines the mechanics of the sensory system. Finding the optimum balance between the drag-torque reception and the hair moment of inertia has been the primary motivation for such optimisation [25].

Taking a closer look at the shape of cercal filiform hairs themselves, could guide towards the first steps of hair shape optimisation. The hairs on the cerci are found to appear in a wide range of lengths from 30 to $1500 \mu \mathrm{m}$ with diameters occurring from 1 to $9 \mu \mathrm{m}$ [10]. Initially, the structural effects of the cercal hairs were analysed by assuming a cylindrical [10] or linearly tapered conical shape [29]. But upon accurate measurements, the hair shape was found to follow extremely elongated paraboloids, i.e. square-root cone, whose diameter increased with the square-root of the distance from their tip [30]. Earlier electron microscope studies showed the hairs to be hollow tubes, with the diameter of the inner hollow cavity being approximately one-third of the outer diameter [31]. The elongated-paraboloid shape of the filiform hairs apparently strikes a fine balance between the drag-torque reception capability and its moment of inertia. The goal is, thus, to fabricate artificial hairs which closely resemble the natural filiform hairs.

B. Artificial hairs: past and present. Artificial hairs were initially fabricated by etching structures on bare silicon wafers and covering them with silicon nitride using low-pressure chemical vapour deposition (LP-CVD). Upon selectively etching the silicon substrate, silicon nitride hairs were fabricated 

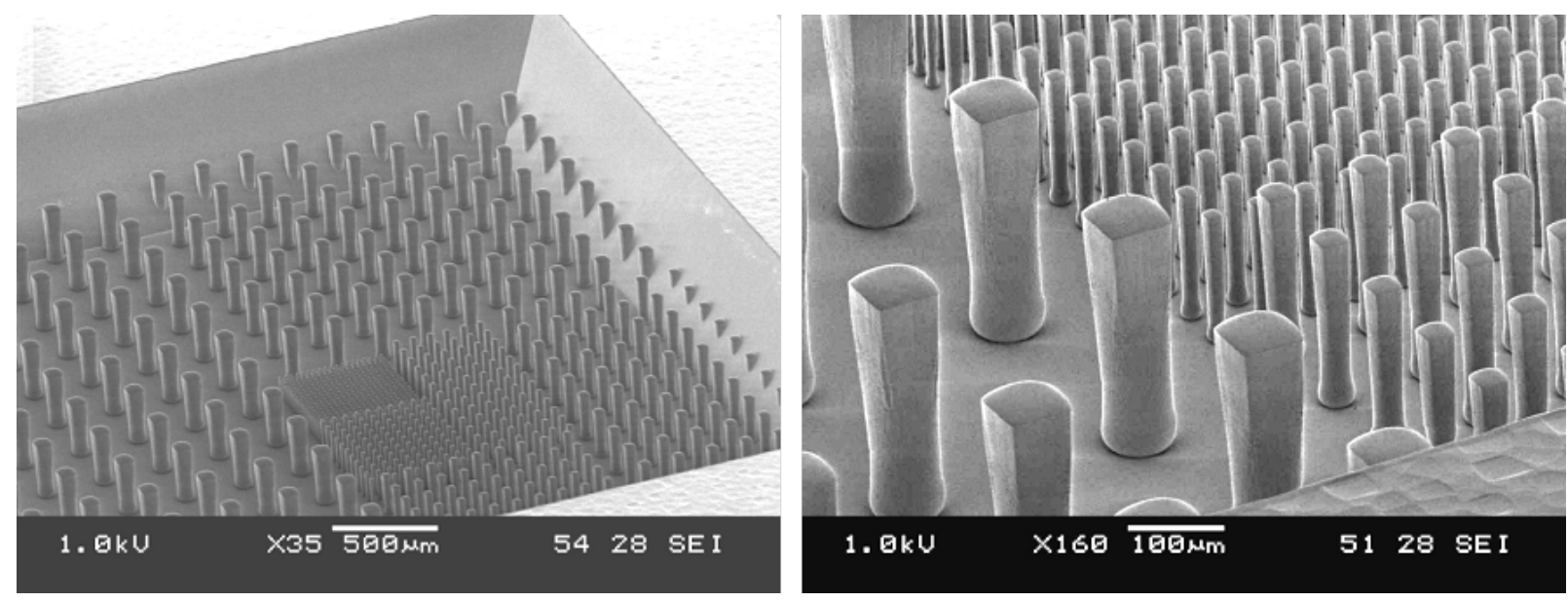

Fig. 5: Initial attempts to make hairs were based on Deep Reactive Ion etching in silicon with subsequent conformal nitride overgrowth and silicon removal.

(Fig. 5) [32]. These hairs were very complex to be integrated into a functional sensor. Subsequent generations of our sensor arrays comprised an artificial hair made of SU-8, a negative-tone, epoxybased photoresist [33], fabricated by means of standard lithography. First, a single layer of SU-8 photoresist was used resulting in hairs of $\approx 450 \mu \mathrm{m}$ length [13] (Fig. 6 left). In later generations of our hair sensors, two subsequent layers of SU-8 were spun and hairs of length of up to $900 \mu \mathrm{m}$ were photo-patterned by top-side exposure [34] (Fig. 6 right). The result was that hairs were long and had a cylindrical shape with a uniform diameter of $\approx 50 \mu \mathrm{m}$.
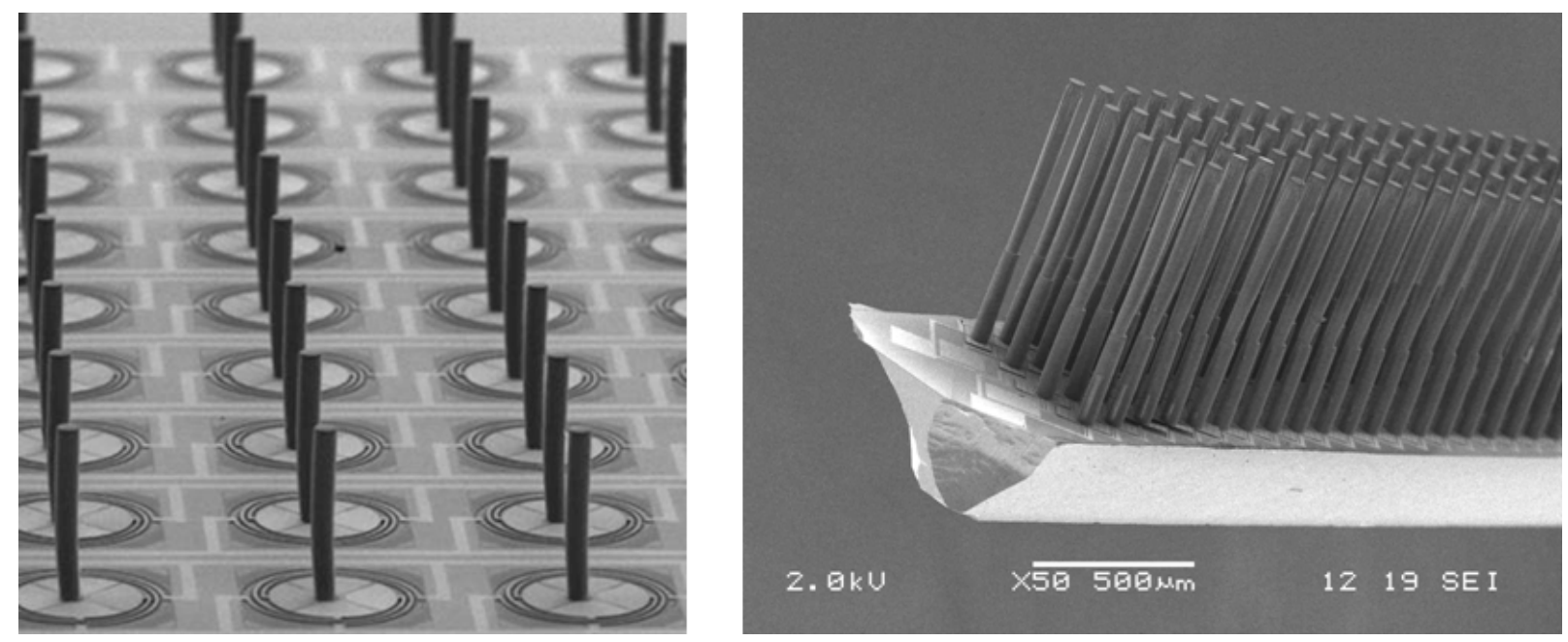

Fig. 6: First (left) and second (right) generation artificial hair-sensors.

For the current generation of artificial hairs (Fig. 3), a new geometry was chosen in order to reduce the hair moment of inertia. The idea is to fabricate artificial hairs in two parts (i.e. two layers of SU-8 photoresist), only that the hair diameter of the top is half as that of the bottom part. Such hair geometry effectively reduces up to $65 \%$ of the hair moment of inertia. One of the difficulties in using multi-spun SU-8 layers for artificial hairs is that alignment of structures on the subsequent layers becomes critical. Further, the standard top-side exposure of SU-8 lithography limits us to achieve hair length variations within an array to only up to 2 or 3 . Therefore, in addition to fabricate artificial hairs with nature-like shape, a new and less-complex fabrication technology is sought in order to realise hairs of varying hair lengths in a wider range, all within the same array.

C. Nature-like hairs: future? Bottom-side exposure of SU-8 layers is a well-known technique, commonly used as molds in the fabrication of micro-needle arrays for drug-delivery applications [35, 36, 
37]. For our requirements, we used the above-mentioned bottom-side exposure for fabricating hairs with gradually tapering tips aimed to resemble the shape of actual filiform hairs of cerci. A simple, proof-of-concept process flow was developed to fabricate SU-8 hairs by the bottom-side exposure method [25]. For the fabrication, a patterned Aluminium layer with circular openings on top of a standard glass substrate is used. Two layers of SU-8 are spun to a thickness of $900-1000 \mu \mathrm{m}$, after which the glass substrate is flipped and exposed through the circular openings. Upon development of the SU-8 layer, nature-like SU-8 hairs, resembling their natural counter-parts closer compared to previous versions of our artificial hairs (Fig. 7 left) were created [25]. Further, the variations in circular opening diameters of the patterned aluminium layer allowed us to achieve a wide range of hair length variations, all in a single step of photolithography (Fig. 7 right) [25].
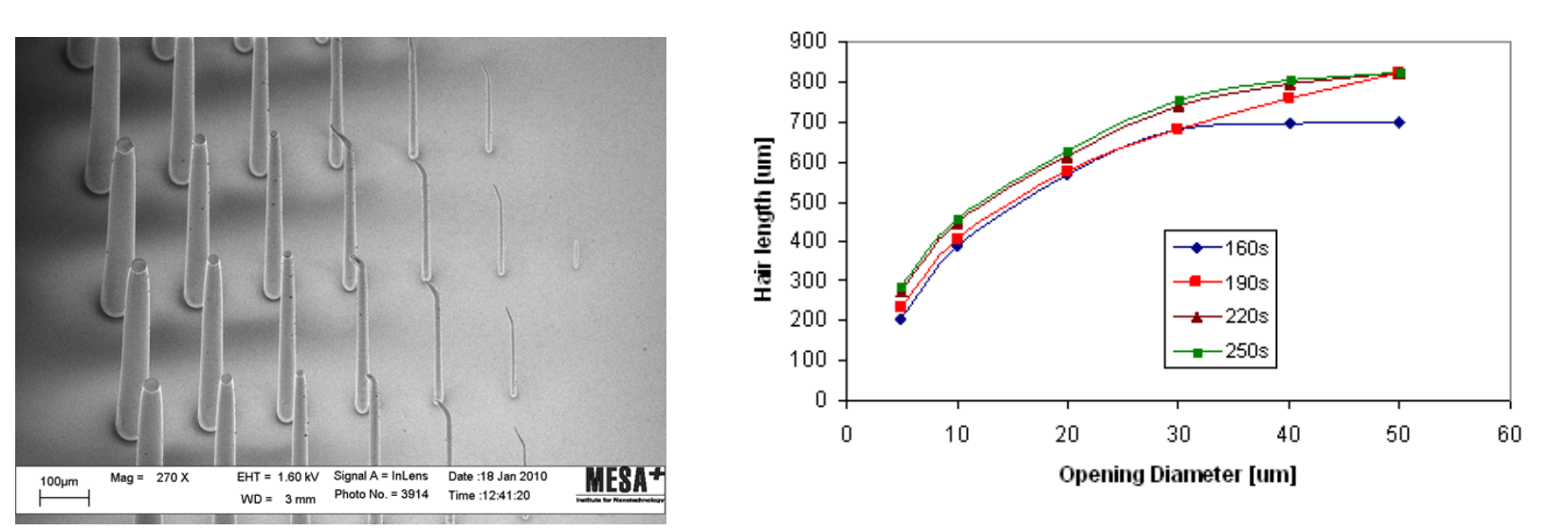

Fig. 7: Nature-like hairs (left) with variations in hair-length determined by the diameter of the open circles used for exposure (right). The process is slightly dependent on illumination time but still sufficiently robust.

The fabricated nature-like hair samples were analysed to find the optimal exposure time and the effect of different design parameters of the aluminium pattern on the hair geometry. The next challenge is to develop a new process scheme to integrate the nature-like SU-8 hairs into the existing sensor fabrication flow. The process flow for wafer-through etch-holes on the silicon wafers for back-side exposure and the applicability of aluminium as both capacitor electrode and hair mask should be tested and optimised.

\section{Viscous coupling}

Arthropods are very often quite hairy, and the high density of flow sensing hairs implies that these hairs interact with each other in order to produce a high resolution map of flow characteristics, acting like a flow camera. The hydrodynamical interactions between hairs, called viscous coupling, have been studied only recently and were found to be highly dependent on the geometrical arrangement of hairs, of their respective lengths and preferential planes of movement, as well as on the frequency content of the input signal [21]. Hairs often interact over long distances, up to 50 times their radius, and usually negatively. Short hairs in particular 'suffer' substantially from the presence of longer hairs nearby. Positive interactions, where the flow velocity at one hair is increased by the presence of nearby hairs, have been however observed in real animals and reproduced computationally. The biological implications of these interactions have only very recently been addressed, and hint towards a coding of incoming signals which relies strongly on the specific sequence of hairs being triggered [22]. In other words, the signature of the incoming signal may be mapped into a given sequence of recruited hairs, which in turn produces a typical sequence of action potentials.

On the physical level it is rather hard to determine viscous coupling effects on real animals due to the pseudo randomness of hair-position, hair-length and hair-orientation. Here the MEMS capabilities 

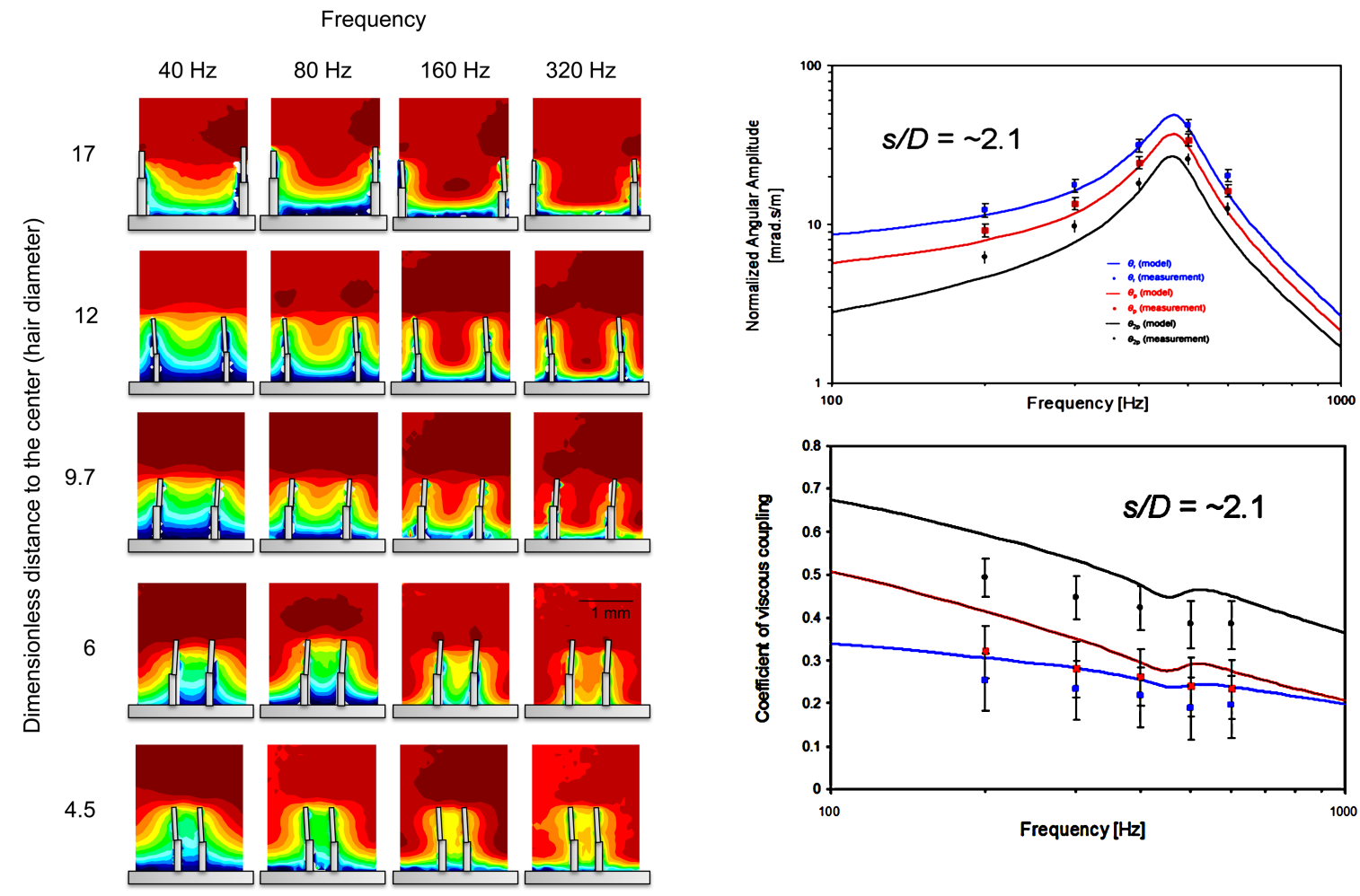

Fig. 8: Spatial harmonic-flow amplitude-distributions for various inter-hair distances and frequencies [21]. Right: Influence of inter-hair viscous coupling on hair-rotation amplitude (top) and viscous coupling coefficient (bottom) versus frequency [24].

to form regular structures with well-defined inter-hair distances present a way to tackle the problem. We have made various structures to systematically investigate viscous coupling effects. Both the flow-profiles [21] as well as the hair-rotations in the presence of perturbing hairs [23, 24] have been studied. An illustration of the effects of shorter inter-hair distances on flow profiles can be seen in Fig. 8, left. The flow-velocities were determined for harmonic flows over a tandem of two MEMSfabricated pillars by particle image velocimetry (PIV). Fig. 8, right - top, shows a frequency response of a hair-sensor with 2 perturbing hairs (black), 1 perturbing hair (red) and no perturbing hair (blue). The hair sensors spacing was $\approx 2.1$ times the hair diameter. Clearly, with one or two perturbing hairs the hair-rotations are smaller than without perturbing hairs. Right - bottom: frequency dependence of the viscous coupling constant for 2 versus 0 perturbing hairs (black), 2 versus 1 perturbing hair (red) and 1 versus 0 perturbing hairs (blue). Lines are predictions based on a modified model introduced in [26] in the limit of arrested hairs, dots are measurements with uncertainty intervals.

\section{Array-sensing}

The SOI based technology not only serves to reduce parasitics but also allows for crossing electrodes since both the silicon device-layer of the SOI wafer as well as the top aluminium layer, mutually separated by silicon-nitride, allow for electrical connections. The technology allows for frequency division multiplexed (FDM) interfacing to individual sensors in a rectangular array, reducing the number of required electrode connections from $3(n \times m)$ to $2 n+m$ for a $n \times m$ array of hair-sensors. Further, this scheme retains the SNR of the hair-sensors at the level they would have had when each single hair-sensor had been individually connected. See Fig. 9 right.

The FDM technique allows for real-time read-out of many sensors in parallel. Therefore it enables the observation of spatio-temporal flow-patterns in which the details carry information of the source of the field, i.e. this type of flow-sensor array in principle allows for the observation (of the movement) of objects in the near-field environment, thus acting as a flow-camera. Fig. 12 left, shows an array of 

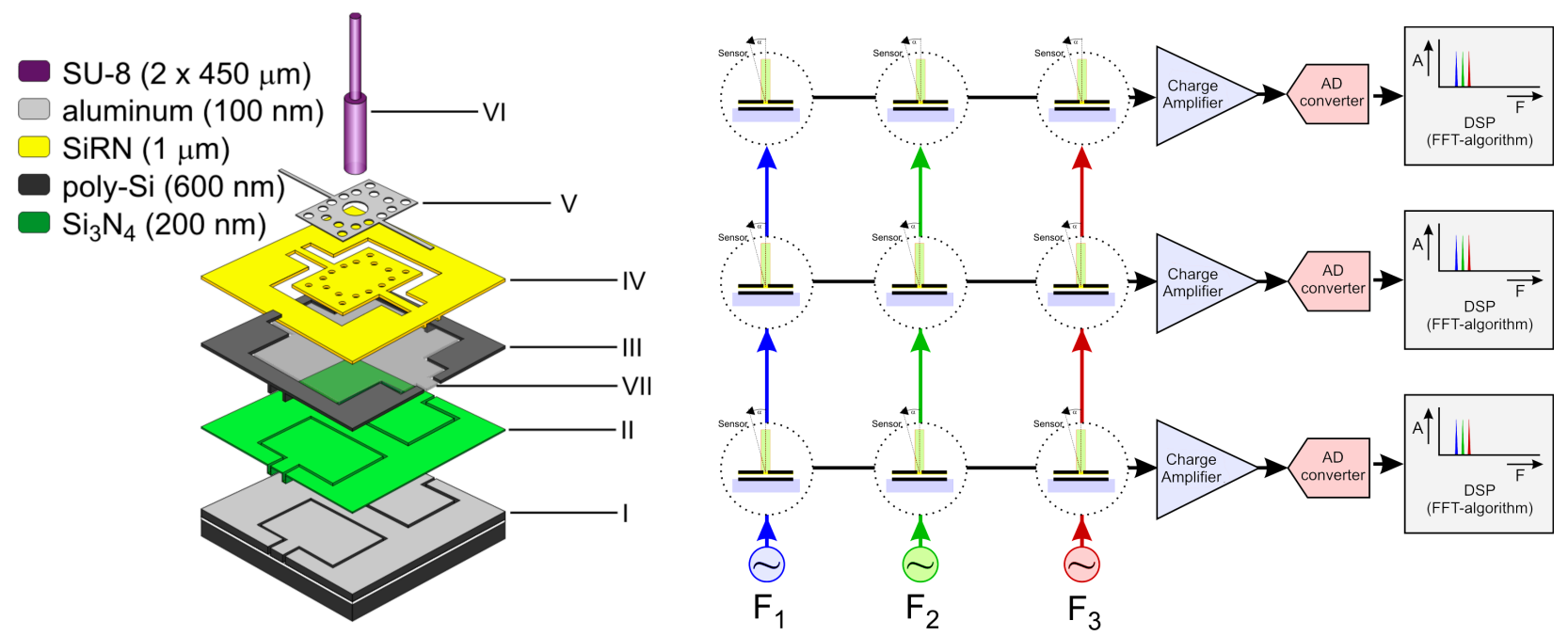

Fig. 9: Left: Extruded SOI-based hair-sensor structure [18]. Right: Frequency Division Multiplexing reduces the number of electrical connections while retaining the original sensor SNR [19].

sensors, each individually interfaced by FDM.

Transient airflow measurements using artificial hair sensors. For many insects airflow patterns, as observed by means of their hair-sensors, carry highly valuable information exposing the sources of these flows. The successful extraction of the characteristics of these spatio-temporal airflow patterns will give us insight in their features and information contained in them. In nature, there are numerous examples representing transient airflow stimuli such as spider motion [41] and (passing) humming flies [38].

In most investigations on our artificial hair-sensors the measurements were conducted using sinusoidal airflows [18]. Obviously, using transient signals spatio-temporal information becomes richer and array-measurements will allow to capture important flow events. Here we describe measurements of spatio-temporal airflow fields generated by a pulsed-like airflow by means of our artificial hairsensor arrays. The measurements show the hair-sensors ability to determine the flow field with sufficient temporal and spatial resolution.
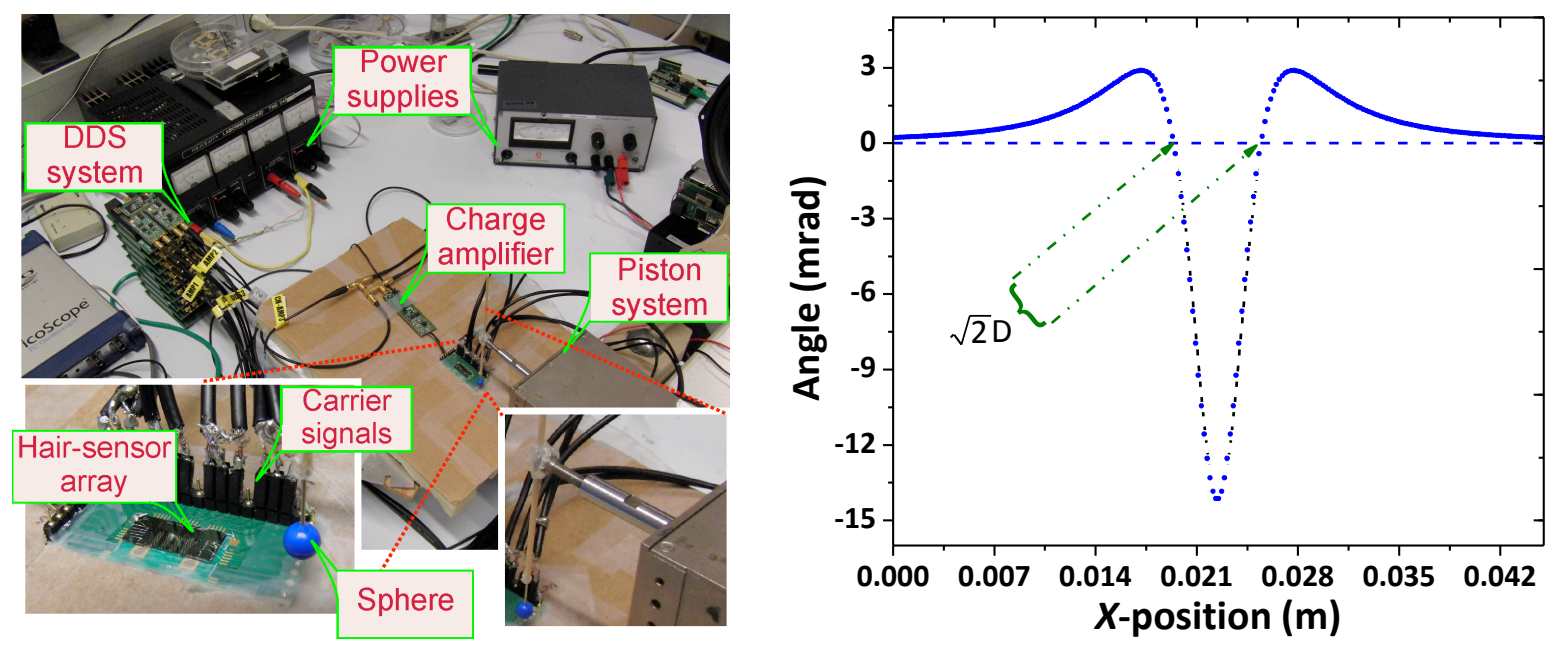

Fig. 10: Left: the setup used for transient measurements. Right: theoretical transient dipole flow-field parallel to the direction of orientation of the flow-sensor. 
Measurements setup and results We measured responses of our biomimetic hair sensors to airflow transients using a sphere with $3 \mathrm{~mm}$ radius attached to a piston system to represent the motion of a spider at a distance $(D)$ from the substrate. The sphere moves parallel to the x-axis. A single-chip array consisting of single hair sensors is used for flow-detection. Fig. 10 left shows a photograph of the measurement setup.

The results show that our hair sensor is able to capture the essential features of the transient airflow field generated by the moving sphere. Interestingly, the hair-sensor response shows strong similarities with the field shape generated by the dipole source. Fig. 10 right and Fig. 11 left show an example of theoretical and measured hair-sensor responses due to the sphere movement. The distance to the sphere is encoded in the characteristic points of the flow-field [39]. Hence, it can be derived from the sensor output. In the transient response the time difference between the characteristic points can be translated into position using the piston speed, and subsequently into an estimated distance $\left(D_{\text {est }}\right)$ between sphere and hair sensor. Fig. 11 right shows $D_{\text {est }}$ versus $D$ using the transient hair response.
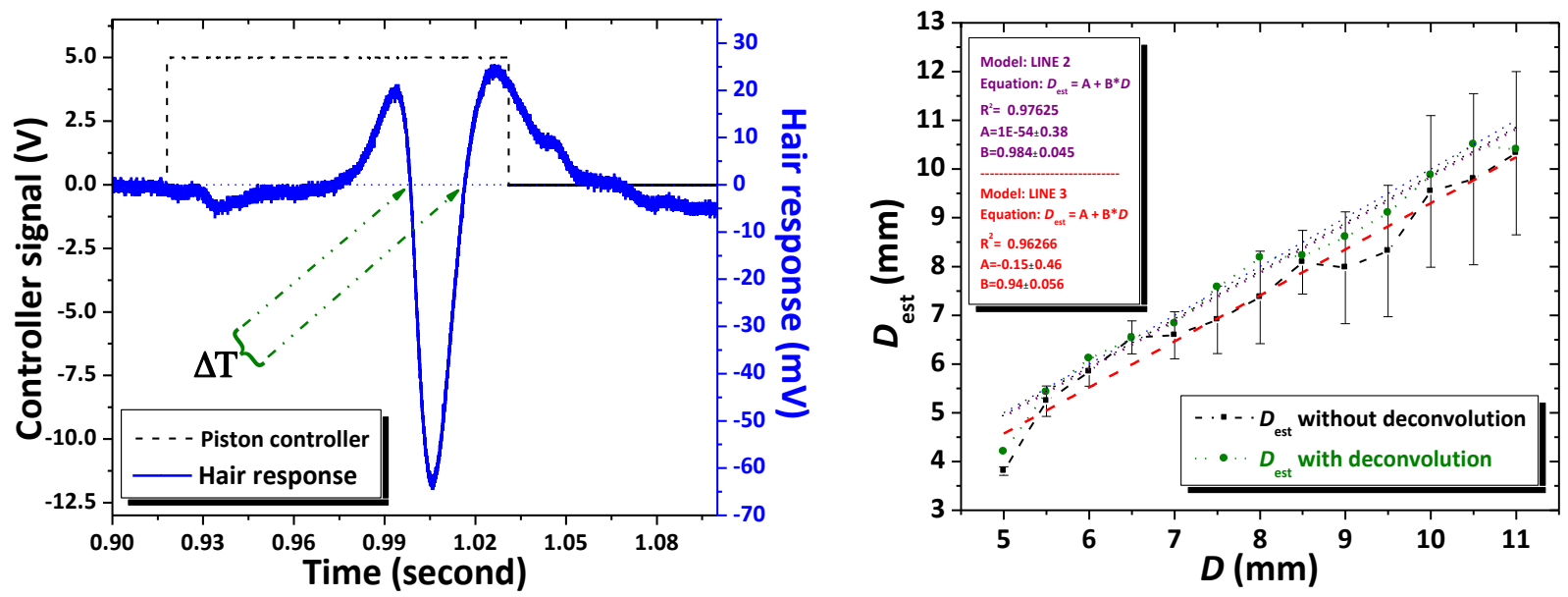

Fig. 11: Left: an example of measured hair sensor response (solid) when exposed to a transient flow. Right: $D_{\text {est }}$ versus $D$ using transient hair-response measurements, before (solid-squares) and after (solid-circles) deconvolving the hair-sensor response. The best linear-line fit for both measurements are compared with ideal linear-line (dotted). $D$ represents the height of the sphere centre relative to the substrate. The error bars represent the uncertainty in determining the zero-crossing points of the measured dipole profile.

To exclude effects of the hair-mechanics a deconvolution was performed to recover the flow-velocity. The results show that the deconvolved sensor data nearly matches the raw sensor data with a slight widening in its characteristic points. Hence, we anticipate that the hair sensor is following the development of the flow profile rather well, a consequence of the nearly critical damped system with best frequency in the range of $250-300 \mathrm{~Hz}$. The results using the deconvolved sensor data (Fig. 11 right) indicate that the linear-line fit of $D_{\text {est }}$ more closely matches the physical $D$ while for the raw sensor data the $D_{\text {est }}$ seems to closer resemble the distance to the centre of the hair shaft. This highlights the effect of the mechanics and the hair-shaft of the sensor. Since we know of no way to correct for the integrated drag-force on the length of the hair we consider the torque as a reasonable representation of the flow field at between $1 / 2$ and $2 / 3$ the hair length (i.e. $\approx 600-700 \mu \mathrm{m}$ above the substrate).

Arrays of hair sensors offer us spatial information, specifically if they are measured simultaneously. Here we integrated Frequency Division Multiplexing (FDM [40]) to simultaneously measure the transient response of multiple hairs i.e. spatio-temporal airflow pattern measurements. Fig. 12 left shows an image of a single-chip hair-sensor and Fig. 12 right shows the response of four single-hair sensors in one row, when they are exposed to a transient airflow produced by a moving sphere. 

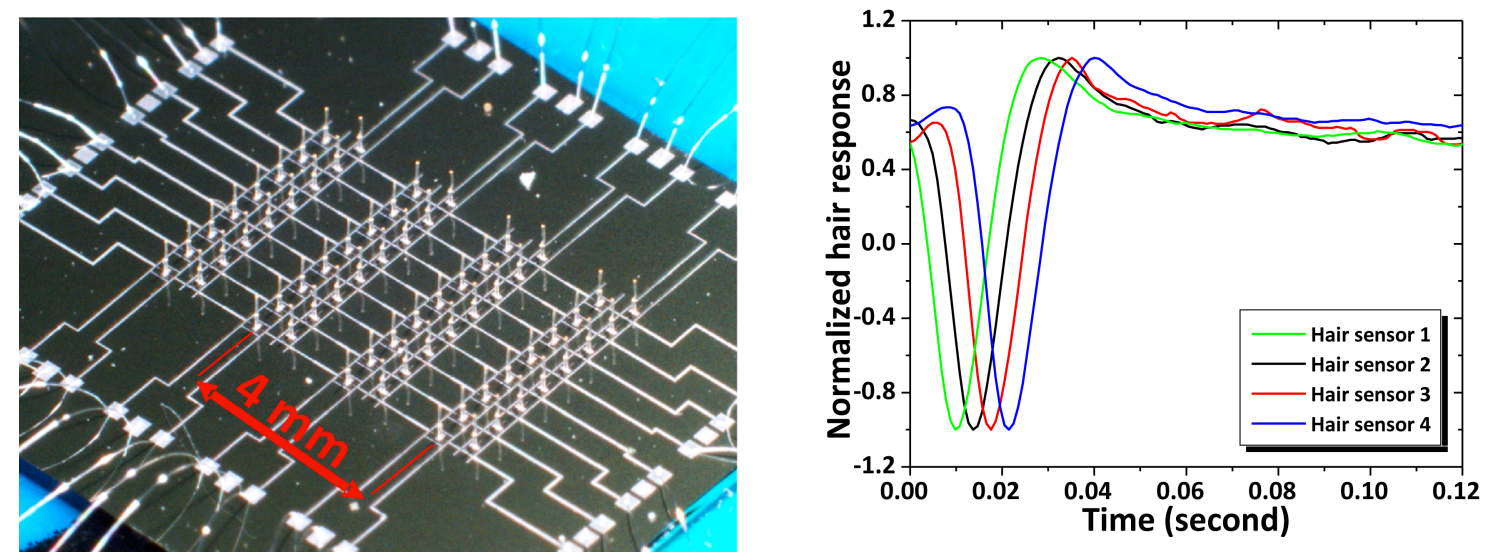

Fig. 12: Left: microphotograph of an $8 \times 8$ array of individually FDM addressable hair-sensors, ordered in pairs with orthogonal directivity [19]. Right: normalized output of 4 simultaneously measured sensors when exposed to a sphere passing by at certain distance [40].

Using the signal profiles as detected by the entire array would allow us to determine an increasing number of source properties. By virtue of the piston velocity the delay represents the separation distance in between two hairs divided by the sphere velocity. Thus, the sphere velocity can be determined independently of the distance to the sphere.

As a first trial, we have been able to detect delays using the signals from four hairs in one row, Fig. 12 left, with sphere propagation speed of $38 \mathrm{~cm} / \mathrm{s}$ positioned at $D=5 \mathrm{~mm}$ with $2 \mathrm{~mm}$ separation distance in between each pair of hairs, while using FDM. The measurements show about $4 \mathrm{~ms}$ time delay between each two subsequent hairs-sensor responses. Fig. 12 right shows the normalised transient response of four single-hair sensors arranged in one row measured with FDM. This demonstrates the possibility to perform spatio-temporal flow pattern measurements using a single-chip hair sensor array with FDM and to, subsequently, use the features of these flow profiles to determine source parameters (i.e. size, speed and position).

Measurements like these, in principle, allow to extract the following information. a) The projection of the velocity of the passing sphere in the direction parallel to the row of the sensor array can be determined using the distance between the sensors and the time of flight. b) Once the velocity is known, the distance of the sphere trajectory perpendicular to the row of sensors can be determined from the characteristic points of the dipole-induced signal [20]. c) With the distance to the sphere and its velocity known, the amplitude of the signal can be used to determine the size of the sphere. d) Additional sensors allow to track the motion of the sphere in other directions as well. As an example Fig. 12 right, shows at the normalised responses for 4 hair-sensors in line where the sphere was moved along. From the sensors-responses a speed of $512 \mathrm{~mm} / \mathrm{s}$ at a distance of $\approx 5-7 \mathrm{~mm}$ can be inferred [20, 40]. Clearly, any of the above does not reflect the way crickets use their hair-sensor arrays but it is instructive to see what information a multitude of sensors in principle can uncover.

In conclusion, the feasibility of artificial hair-sensor arrays to measure spatio-temporal flow patterns has been demonstrated, opening up the possibility to develop high-resolution flow cameras. As an example, we mimicked the cricket-spider escape mechanism using a moving sphere to generate spatiotemporal airflow patterns i.e. transient airflow. The response of single hair-sensors within an array was measured simultaneously using FDM array interfacing. Performing source localisation using the measured signals demonstrates how far we can go with our hair-sensors, either as single sensor or in arrays to detect spatio-temporal airflow fields. The above results also can shed some light upon the mechanisms that are at work in crickets' flow sensing and how they might exploit spatio-temporal airflow patterns to estimate the occurrence and direction of moving objects in their close environment 
(both of which have been described in literature [41]).

\section{Beyond bio-inspiration: parametric effects}

Apart from using the capacitively interrogated hair-sensors strictly for sensing, one may achieve parametric effects by application of additional DC or AC bias-voltages to the electrodes (Fig. 13 left). These voltages will produce electrostatic forces, which in a balanced situation (i.e. no tilt of the hair) do not change the rotational angle, but in a tilted situation will produce the largest forces on the side with the smallest gap. So, the electrostatic torque tends to add to the flow induced torque and therefore the applied voltages will serve as an electronic means to adaptively change the spring-stiffness of the hair-sensor system, i.e. electrostatic spring softening (ESS).
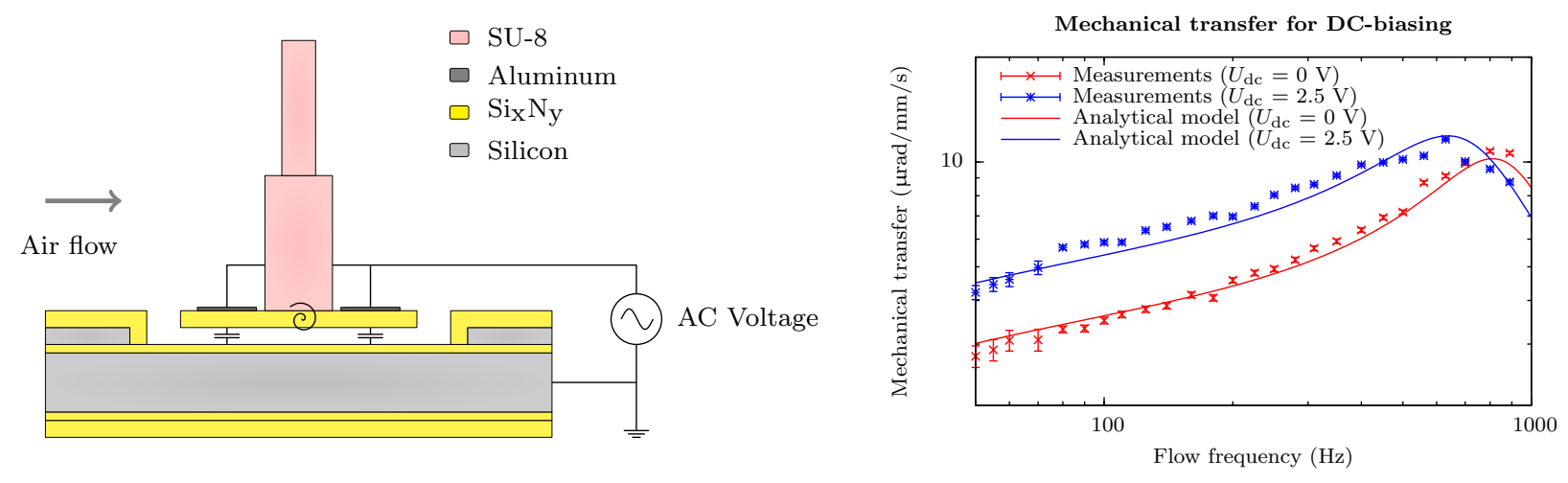

Fig. 13: Left: electrodes can also be exploited for electrostatic actuation. Right: improvement of the mechanical responsivity and reduction of the resonance frequency on DC-bias induced ESS [27].

DC-biasing. A DC-bias voltage can be used to change the system's torsional stiffness. Experimentally the mechanical transfer is determined for flow frequencies from 100 to $1000 \mathrm{~Hz}$ with and without the application of a DC-bias voltage. During this measurement, a DC-bias voltage $U_{\mathrm{dc}}$ of $2.5 \mathrm{~V}$ is used, giving an increase in sensitivity of about $80 \%$ for frequencies within the sensor's bandwidth. Also lowering of the resonance frequency $\omega_{r}$ is observed (about 20\%). Overall, measurements are in good agreement with modelling and it is shown clearly that DC-biasing leads to a larger sensitivity below the sensor's resonance frequency (Fig. 13 right) [16].

Parametric amplification. To improve the performance of these sensors even further and implement adaptive filtering, we make use of non-resonant parametric amplification (PA). Parametric amplification is a mechanism based on modulation of one or more system parameters, in order to control the system behavior. This leads to complex interactions between the modulating signals in which amplitude, frequency and phase play important roles [42]. In this work, we obtain the conditions for PA by changing the DC-bias voltage to an AC-bias voltage (also called pump signal), which is another way of exploiting ESS.

Parametric amplification can give selective gain or attenuation, depending on the pump frequency $f_{\mathrm{p}}$ and pump phase $\phi_{\mathrm{p}}$. Equal frequencies for flow and pump $\left(f_{\mathrm{p}}=f_{\mathrm{a}}\right)$ give coherency in torque and spring softening, for which the pump phase determines whether the system will show relative amplification or attenuation. Therefore, it is possible to realize a very sharp band pass/stop filter, depending on the pump settings.

Setting the frequency of the AC-bias voltage to $150 \mathrm{~Hz}$, its amplitude to $5 \mathrm{~V}$ and the pump phase to the value producing maximum gain, and supplying an oscillating air flow consisting of three frequency components $(135 \mathrm{~Hz}, 150 \mathrm{~Hz}$ and $165 \mathrm{~Hz}$ ), filtering and selective gain of the flow signal are 
demonstrated. The presence of a bias-signal, through the action of non-resonant PA, increases the frequency-matched signal by $20 \mathrm{~dB}$, whereas the other two components are only amplified by $8-9 \mathrm{~dB}$, resulting in selective gain of the flow signal [28].
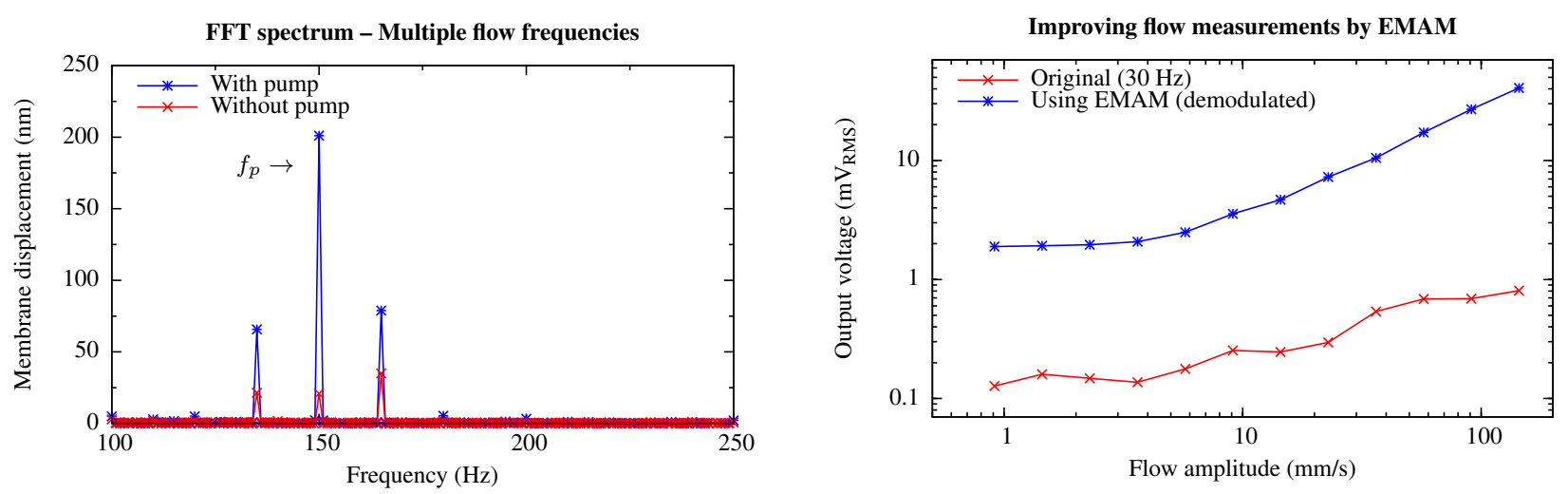

Fig. 14: Left: Measured gain of about $20 \mathrm{~dB}$ for the flow frequency component at $150 \mathrm{~Hz}$ determined by FFT. The AC-bias voltage is fixed at $f_{\mathrm{p}}=150 \mathrm{~Hz}$ with an amplitude of $5 \mathrm{~V}$. Right: Improvement of the quality of the measured RMS-voltage values at low frequency signals using EMAM. In case of EMAM, a clear linear relationship between flow and output voltage is observed above the system's noise level $(>5 \mathrm{~mm} / \mathrm{s})$.

ЕМАМ. We also implemented ESS by setting the AC-bias voltage frequency considerably higher than the frequency of the air flow. As a result, the system's spring-stiffness is electromechanically modulated, which results in Electro Mechanical Amplitude Modulation (EMAM). Experimentally, generating a harmonic flow at $30 \mathrm{~Hz}$ and setting the AC-bias voltage frequency to $300 \mathrm{~Hz}$, the flow is modulated and the flow information is upconverted to higher frequencies [44].

The incoming air flow signal is recovered by demodulation (using synchronous detection) of the measured rotational angle. Without EMAM, a noisy relationship between the flow amplitude and the resulting output voltage is observed. Also, large, undesired, fluctuations are observed (Fig. 14 right). However, with EMAM, a clear linear relationship is observed for flow velocity amplitudes above $5 \mathrm{~mm} / \mathrm{s}$, showing that the measurement quality of low frequency flows too can be improved by ESS.

\section{Summary and conclusions}

Crickets possess a sensitive, distributed hair-sensor system with near to thermal-mechanical noisethreshold sensitivities and which forms an interesting example system for sensory-system engineering. Engineers and biologists working together on this system have been able to make artificial hair-sensor systems and quantify the effects of viscosity mediated coupling. Interfacing arrays of sensors by means of FDM has delivered systems with simultaneous read-out of many sensors and which can be used as flow-cameras. The capacitive structure for read-out doubles as a means for actuation of the hair-sensors and allows such exciting things as parametric amplification and filtering, adaptive - reversible sensormodifications and electromechanical amplitude modulation (frequency shifting of signals). Future work will also encompass studies on the use of stochastic resonance and application of our technology to other bio-inspired sensing modalities.

Despite all advancements in artificial hair-sensor systems the biological example is still is far more complex, evolved and capable. E.g. the full 3-dimensional shape of the cricket cerci, the large number of innervated hairs, the robust generation of neural signals and subsequent intricate processing in the TAG are still far from feasible in current technology. And even if this were technologically possible 
still many things of the cricket flow-sensing system are unknown holding both challenges and promises for the future

\section{Acknowledgements}

The authors would like to thank STW/NWO for funding this research in the framework of the Vici project BioEARS and the EU for funding the Cicada and Cilia projects. Contributions from T. Lammerink and R. Wiegerink have been invaluable. T. Steinmann, E. Berenschot, M. de Boer, R. Sanders and $H$. van Wolferen have given technical support without which this work would not have existed. Numerous students have contributed to this research, for which they are gratefully acknowledged.

\section{References}

[1] T. Shimozawa, J. Murakami and T. Kumagai, Cricket wind receptors: thermal noise for the highest sensitivity known. In: Sensors and sensing in biology and engineering (eds F. G. Barth, J. A. Humphrey \& T. W. Secomb), Springer, Berlin, 2003, pp. 145-159.

[2] J.A. Humphrey and F.G. Barth, Medium flow-sensing hairs: biomechanics and models. In: Advances in insect physiology. Insect mechanics and control, vol. 34 (eds J. Casas \& S. J. Simpson), Elsevier, Amsterdam, 2008, pp. 1-80.

[3] J. P. Miller, S. Krueger, J. J. Heys, T. Gedeon, Quantitative Characterization of the Filiform Mechanosensory Hair Array on the Cricket Cercus, PLoS ONE 6(11): e27873. PLoS ONE 7(1): e30115. doi:10.1371/journal.pone.0027873.

[4] Jeffrey J. Heys, Prathish K. Rajaraman, Tomas Gedeon and John P. Miller, A Model of Filiform Hair Distribution on the Cricket Cercus, PLoS ON E 7(10): e46588. doi:10.1371/ journal.pone.0046588.

[5] Olivier Dangles, Thomas Steinmann, Dominique Pierre, Fabrice Vannier and JérômeCasas, Relative contributions of organ shape and receptor arrangement to the design of cricket $\square \mathrm{s}$ cercal system, J. Comp. Physiol. A (2008) 194:653-663, doi:10.1007/s00359-008-0339-x.

[6] Jacobs, G.A., Miller, J.P., Aldworth, Z.: Computational mechanisms of mechanosensory processing in the cricket. J. Exp. Biol. 211, 1819-1828 (2008).

[7] H. Lamb, The dynamical theory of sound, Edward Arnold, London, 1910.

[8] R. Kant and J. A. C. Humphrey, Response of cricket and spider motion-sensing hairs to airflow pulsations, J. R. Soc. Interface, 2009, doi:10.1098/rsif.2008.0523.

[9] J. Tautz and H. Markl, Caterpillars Detect Flying Wasps by Hairs Sensitive to Airborne Vibration, Behav. Ecol. Sociobiol. 4, 101-110 (1978).

[10] J. Humphrey, R. Devarakonda, I. Iglesias, F. Barth, Dynamics of arthropod filiform hairs. I. Mathematical modeling of the hair and air motions, Phil. Trans.: Bio. Sc. 340, 423-444, 1993.

[11] T. Shimozawa, T. Kumagai and Y. Baba, Structural scaling and functional design of the cercal windreceptor hairs of cricket, J. of Comp. Physiol. A, Vol. 183, pp. 171-186, 1998.

[12] Stokes, G.G. 1851, On the effect of the internal friction of fluids on the motion of pendulums, Transactions of the Cambridge Philosophical Society, Vol. IX. p. 8, reprinted in Mathematical and Physical Papers, vol. 111, pp. 1-141, Cambridge University Press, 1901. 
[13] M. Dijkstra, J. van Baar, R. Wiegerink, T. Lammerink, J. de Boer and G. Krijnen, Artificial sensory hairs based on the flow sensitive receptor hairs of crickets, J. Microm. Microeng. 15, S132-S138, 2005.

[14] R. Jaganatharaja, C. Bruinink, B. Hagedoorn, M. Kolster, T. Lammerink, R. Wiegerink and G. Krijnen, Highly-sensitive, biomimetic hair sensor arrays for sensing low-frequency air flows, proc. Transducers 2009, Denver, USA, 21-25 June 2009, pg 1541-1544.

[15] M. McConney, C. Schaber, M. Julian, F. Barth, V. Tsukruk, Visco-elastic nanoscale properties of cuticle contribute to the high-pass properties of spider vibration receptor (Cupiennius salei Keys), J. Royal Soc. Interface, Vol 4, 2007, p1135-1143, doi:10.1098/rsif.2007.1000

[16] H. Droogendijk et. al, in preparation.

[17] G. Krijnen, T. Lammerink, R. Wiegerink and Jérôme Casas, Cricket Inspired Flow-Sensor Arrays, Proc. IEEE Sensors 2007, Atlanta, p539-546.

[18] A. Dagamseh, C. Bruinink, H. Droogendijk, R. Wiegerink, T. Lammerink, and G. Krijnen, Engineering of biomimetic hair-flow sensor arrays dedicated to high-resolution flow field measurements, Proceedings IEEE-Sensors 2010, November 1-4, 2010, Waikoloa, Hawaii, USA, pg 2251 $-2254$.

[19] A. Dagamseh, T. Lammerink, R. Sanders, R. Wiegerink, G. Krijnen, Towards high-resolution flow cameras made of artificial hair flow-sensors for flow pattern recognition, 24th IEEE MEMS 2011, January 23-27, 2011, Cancun, Mexico. pp. 648-651.

[20] J. Franosch, A. Sichert, M. Suttner, J. Van Hemmen, Estimating position and velocity of a submerged moving object by the clawed frog Xenopus and by fish - A cybernetic approach, Biological Cybernetics, 93, 2005, pp. 231-238.

[21] J. Casas, T. Steinmann and G. Krijnen, Why do insects have such a high density of flow-sensing sensors hairs? Insights from the hydromechanics of biomimetic MEMS, J. R. Soc. Interface 7, 2010, 1487-1495.

[22] J. Mulder-Rosi, G.I. Cummins, J.P. Miller, The Cricket Cercal System Implements Delay-Line Processing, J. Neurophysiology, Volume: 103, Issue: 4, 2010, Pages: 1823-1832.

[23] R. Jaganatharaja, H. Droogendijk, S. Vats, B. Hagedoorn, C. Bruinink, G. Krijnen, Unraveling the viscosity-mediated coupling effect in biomimetic hair sensor arrays. In: 24th IEEE Int. Conf. on Micro Electro Mechanical Systems, 2011, 23-27 Jan 2011, Cancun, Mexico.

[24] Jaganatharaja et. al. Paper in preparation.

[25] R. Kottumakulal, C.M. Bruinink and G.J.M. Krijnen, in preparation.

[26] B. Bathellier, F. Barth, J. Albert, J. Humphrey, Viscosity-mediated motion coupling between pairs of trichobothria on the leg of the spider Cupiennius salei, J Comp. Physiol. A (2005) 191: 733 - 746, doi:10.1007/s00359-005-0629-5.

[27] H. Droogendijk, C. Bruinink, R. Sanders, A. Dagamseh, R. Wiegerink and G. Krijnen, Improving the performance of biomimetic hair-flow sensors by electrostatic spring softening, J. Micromech. Microeng. 22 (2012) 065026, doi:10.1088/0960-1317/22/6/065026.

[28] H. Droogendijk, C. Bruinink, R. Sanders, and G. Krijnen, Non-resonant parametric amplification in biomimetic hair flow sensors: Selective gain and tunable filtering, Applied Physics Letters 99, 213503 (2011), doi:10.1063/1.3663865. 
[29] T. Shimozawa, M. Kanou, The aerodynamics and sensory physiology of range fractionation in the cercal filiform sensilla of the cricket Gryllus bimaculatus, J. Comp. Physiol. A (1984) 155, pp. 495-505.

[30] T. Shimozawa, T. Kumagai, Y. Baba, The shape of wind-receptor hairs of cricket and cockroach, J. Comp. Physiol. A (1998) 183, pp. 171-186.

[31] W. Gnatzy, Development of the Filiform hairs on the Cerci of Gryllus bimaculatus Deg. (Saltatoria, Gryllidae), Cell Tiss. Res. (1978) 187, pp. 1-24.

[32] J.J. van Baar, M. Dijkstra, R.J. Wiegerink, T.S.J. Lammerink and G.J.M. Krijnen, Fabrication of arrays of artificial hairs for complex flow pattern recognition, Proceedings of IEEE Sensors conference, Toronto, Canada, October 22-24, 2003.

[33] http://www.microchem.com/products/su_eight.htm.

[34] S.S. Siripurapu, Cricket-inspired sensory hairs with capacitive motion detection, Master thesis, University of Twente (2005).

[35] H-C. Huang and C-C. Fu, Out-of-plane polymer hollow micro needle array integrated on a microfluidic chip, Proc. of IEEE Sensors, Irvine, USA, 2005, pp. 484-487.

[36] K. Kim, D.S. park, H.M. Lu,W. Che, K. Kim, J-B. Lee and C.H. Ahn, A tapered hollow metallic microneedle array using backside exposure of SU-8, J. Micromech. Microeng, (2004) Vol.14, pp. 597-603.

[37] H. Yu, K. Shibata, B. Li and X. Zhang, Fabrication of a hollow metallic microneedle array using scanning laser direct writing, Proc. of 9th Int. Conf. on Miniaturized systems for chemistry and life sciences, Boston, USA, Oct 9-13, 2005, pp. 187-189.

[38] Barth, F.G., Humphrey, J.A., Wastl, U., Halbritter, J. and Brittinger, W. (1995), Dynamics of arthropod filiform hairs. III. Flow patterns related to air movement detection in a spider (Cupiennius salei KEYS.), Philosophical Transactions of the Royal Society B: Biological Sciences, 347, pp. 397-412.

[39] Dagamseh, A.M.K., Lammerink, T.S.J., Kolster, M.L., Bruinink, C.M., Wiegerink, R.J. and Krijnen, G.J.M. (2010) Dipole-source localization using biomimetic flow-sensor arrays positioned as lateral-line system, Sensors and actuators A: Physical, 162, pp. 355-360.

[40] Dagamseh, A.M.K, Wiegerink R. J., Lammerink T.S.J and Krijnen G.J.M. (2012) Towards a high-resolution flow camera using artificial hair sensor arrays for flow pattern observations, Bioinspir. \& Biomim. 7, 046009.

[41] Dangles, O., Ory, N., Steinmann, T., Christides, J. P. and Casas, J. (2006) Spider's attack versus cricket's escape: velocity modes determine success, Anim. Behav., 72, pp. 603-610.

[42] D. Rugar and P. Grütter, Mechanical parametric amplification and thermomechanical noise squeezing, Phys. Rev. Lett., Vol. 67, pp. 699--702, 1991.

[43] M. J. Thompson and D. A. Horsley, Parametrically Amplified Z-Axis Lorentz Force Magnetometer, J. Micromech. Eng., Vol. 20, pp. 702--710, 2011.

[44] H. Droogendijk, C. M. Bruinink, R. G. P. Sanders and G. J. M. Krijnen, Application of electro mechanical stiffness modulation in biomimetic hair flow sensors, Proc., MEMS 2012 Conf., Paris, France, Jan. 29-Feb. 2, 2012, pp. 531-534. 
[45] Bathellier B, Steinmann T, Barth FG, Casas J (2012), Air motion sensing hairs of arthropods detect high frequencies at near-maximal mechanical efficiency, J. R. Soc. Interface 9 71: 11311143. 